\title{
Apical Root Resorption After Orthodontic Treatment
}

\author{
Irinel Panainte, DMD \\ Claudia - Georgeta Grancea, DMD, Postgraduate Student \\ Valentina - Tamara Zamfir - Buta, DMD, PhD Student \\ Mariana Pacurar, Prof., DMD, PhD \\ Department of Orthodontics, Faculty of Dental Medicine, University of \\ Medicine and Pharmacy Targu Mures, Romania
}

doi: 10.19044/esj.2016.v12n24p43 URL:http://dx.doi.org/10.19044/esj.2016.v12n24p43

\begin{abstract}
Aim of the study: to find if apical root resorption is related to orthodontic treatment time, type of appliance used and which are the most susceptible teeth to develop this type of resorption. Material and methods: 70 patients (27 males and 43 females) selected from patients reffered for an orthodontic treatment at the Orthodontic Department of Faculty of Dentistry, University of Medicine and Pharmacy from TirguMures. The mean age at the beginning of treatment was 15.11 years for males and 14.67 years for females subjects. At the end of treatment, on their panormaic radiographs it was measured apical root resorption on incisors, premolars and molars in the upper and lower arch. Results: Root resorption was significantly $(p<0.05)$ correlated with fixed appliance treatment (49 percent). Patients with the longest treatment periods presented with significantly $(\mathrm{p}<0.05)$ more grade 2 resorption: 28 months ( $\pm 2.6 \mathrm{SD})$ in the upper arch and 30 months ( $\pm 3.2 \mathrm{SD})$ in the lower arch. In the patients with the lowest treatment period (16 months in the maxilla and 18 months in the mandible arch) it was found no resorption. In the upper arch most of the patients (22.22 \% males and 18.6\% females) showed a grade 2 resorption in the incisor area. Root resorption of the premolars was seen in $18.5 \%$ of the male patients (7.4\% with grade 2 ) and in $16.26 \%$ of the female patients (6.97 with grade 2). Conclusions: There is a high correlation between the orthodontic treatment time and apical root resorption. Most exposed to this process are incisors from bot, upper and lower arch. Less resorption was noticed in the premolar area.
\end{abstract}

Keywords: Orthodontic treatment, root, apical resorption, fixed appliance 


\section{Introduction}

When we talk about root resorption, we understand the loss of substance or bone by physiological or pathological means, such as the reduction of the volume and size of the residual ridge of the mandible or maxilae and also, that the cementoclastic and dentionoclastic action that may occur on a tooth root (Al-Qawasmi et al 2003).

Nowadays, need for orthodontic treatment has increased. This is why, many patients are looking to be treated, especially when they are in adolescence years. Unfortunately with all of these comes a series of undesirable iatrogenic problems. One of the most common iatrogenic situation is the apical root resorption associated with the orthodontic treatment (Apajalahti et al 2007). From a certain point of view, it becomes very important, since this secundar effect can not be prevented in those situations when it occurs. Because of this reasons, many researchers were intrested in this subject, but still controversy exists regarding its causes and predisposing factors (Hartsfield et al 2004, Segal et al 2004).

There are a lot of factors supposed to be involved in this process, but no one could say for sure the precise causes for its development (Liou et al 2010, Dudic et al. 2009). From this point of view, this study's purpose is to find if apical root resorption is related to orthodontic treatment time, type of appliance used and which are the most susceptible teeth to develop this type of resorption.

\section{Materials and methods}

After the research protocol was established, it was approved by the Ethical Commitee of Scientific Research of the University of Medicine and Pharmacy from TirguMures. A written consent was obtained from each patient or their parents in order to use patient's radiograph for this study.

In the study were included 70 patients (27 males and 43 females) selected from patients reffered for an orthodontic treatment at the Orthodontic Department of Faculty of Dentistry, University of Medicine and Pharmacy from TirguMures. The mean age at the beginning of treatment was 15.11 years for males and 14.67 years for females subjects (Table 1). Most patients (84 percent) had more then one type of appliance during treatment (Table 2).

$\begin{array}{cc}\text { AGE (YEARS) } & \text { MALE } \\ 12 & 3 \\ 13 & 4 \\ 14 & 2 \\ 15 & 4 \\ 16 & 6 \\ 17 & 8 \\ \text { TOTAL } & 27 \\ \text { MEAN AGE } & 15.11\end{array}$
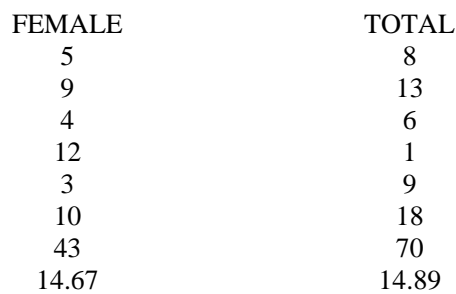

Table1 Age distribution of orthodontically treated patients at the beginning of treatment 


$\begin{array}{cccc}\text { APPLIANCE } & \mathrm{N} & \% & \begin{array}{c}\text { Mean duration of } \\ \text { treatment (SD) }\end{array} \\ \text { Fixedappliance } & 46 & 65.7 & 1.8( \pm 1.0) \\ \text { Active removableplates } & 21 & 30 & 1.6( \pm 1.4) \\ \text { Activator } & 19 & 27.14 & 2.1( \pm 1.2) \\ \text { Headgear } & 25 & 35.71 & 1.9( \pm 1.1)\end{array}$

Table2 The type and the mean duration of treatment in years in the orthodontic patients

The digital radiographic images were performed with Pax Flex 3D, Vatech X-ray machine, the exposure time was 12.7 seconds, at $80 \mathrm{KVp}$ and $9.0 \mathrm{~mA}$. The degree of root resorption was evaluated form pre- and posttreatment panoramic radiographs taken on average 6 months after the end of treatment.

The inclusion criteria were: patients with age between 12 and 17 years, treatment time at least 18 months, good radiograph images before and after treatment. The patients with panoramic radiographs in which the roots were distorted and not clearly visible were rejected. The post-treatment root lenghts of all tooth group, except third molars, were compared with the root lenghts on the pretreatment radiographs. The degree of root resorption was assessed using grades from 0 to 2 . In order to determine differences in the prevalence of root resorption between genders a chi-square test was used.

\section{Results}

Nine male patients (33.33\%) and sixteen (37\%) females developed root resorption. Root resorption was significantly $(\mathrm{p}<0.05)$ correlated with fixed appliance treatment (49 percent). Patients with the longest treatment periods presented with significantly $(\mathrm{p}<0.05)$ more grade 2 resorption: 28 months ( $\pm 2.6 \mathrm{SD})$ in the upper arch and 30 months $( \pm 3.2 \mathrm{SD})$ in the lower arch. In the patients with the lowest treatment period (16 months in the maxilla and 18 months in the mandible arch) it was found no resorption (Table 3).

\begin{tabular}{|c|c|c|c|c|}
\hline \multicolumn{5}{|c|}{ Mean treatment time in months $( \pm \mathrm{SD})$ Group total } \\
\hline & & 0 & 1 & 2 \\
\hline \multicolumn{5}{|c|}{ Fixedappliance treatment } \\
\hline Maxilla & $16( \pm 2.5)$ & $23( \pm 3.2)$ & $28( \pm 2.6)$ & $20( \pm 4.6)$ \\
\hline Mandible & $18( \pm 4.3)$ & $25( \pm 3.5)$ & $30( \pm 3.2)$ & $24( \pm 3.8)$ \\
\hline
\end{tabular}

Table 3 Mean treatment duration in months( \pm standard deviation) with fixed appliance in relation with resorption grade

From all analyzed teeth, the highest rate of resorption was found in the maxillary and mandibular incisors (Table 4). In the upper arch most of the patients (22.22 \% males and $18.6 \%$ females) showed a grade 2 resorption in the incisor area. The lowest rate of apical root resorption was noticed for bicuspids, both in the upper arch and in the lower arch, both males and 
females subjects. Root resorption of the premolars was seen in $18.5 \%$ of the male patients (7.4\% with grade 2 ) and in $16.26 \%$ of the female patients (6.97 with grade 2). No significant differences in the degree of root resorption were found between the malocclusion classes or other pretreatment features.

\begin{tabular}{llclc} 
& \multicolumn{2}{c}{ Number of males (\%) } & \multicolumn{2}{c}{ Number of female(\%) } \\
& Grade 1 & $\begin{array}{c}\text { Grade 2 } \\
\text { Maxilla }\end{array}$ & Grade 1 & Grade 2 \\
Incisors & $5(18.25)$ & $6(22.22)$ & $7(16.27)$ & $8(18.6)$ \\
Canines & $3(11.11)$ & $2(7.4)$ & $4(9.3)$ & $3(6.97)$ \\
Premolars & $2(7.4)$ & $1(3.7)$ & $1(2.32)$ & $2(4.65)$ \\
Molars & $2(7.4)$ & $1(3.7)$ & $3(6.97)$ & $2(4.65)$ \\
& \multicolumn{5}{c}{ Mandible } \\
Incisors & $4(14.8)$ & $5(18.25)$ & $7(16.27)$ & $9(20.93)$ \\
$\quad$ Canines & $2(7.4)$ & $3(11.11)$ & $2(4.65)$ & $4(9.3)$ \\
Premolars & $1(3.7)$ & $1(3.7)$ & $3(6.97)$ & $1(2.32)$ \\
Molars & $3(11.11)$ & $2(7.4)$ & $4(2.32)$ & $1(2.32)$
\end{tabular}

Table 4 Number and percentage of patients presenting with grade 1 or grade 2 post-treatment apical root resorption in different tooth groups

\section{Discussion}

Although it is not a desirable condition, apical root resorption can occur in orthodontically treated patients, resulting in a permanent loss of substances from the tooth structure in apical area. Since in the last decades the intrest in orthodontic treatment has increased, due to increasing number of dental and skeletal anomalies, same time there is a big amount of side effects of orthodontic therapy, and this type of resorption is one of them (McNab et al 2000).

Because of this, many researchers conducted a lot of studies in order to find out what causes are for the development of this process. In one of their studies, Brezniak and Wasserstein tried to conclude about the most common factors associated with increased levels of apical root resorption : biological, mechanical and a combination of biological and mechanical factors (Brezniak et al 2002)

Biologic conditions as endocrine disorders, nutritional problems or hormonal imbalances, were cited sometimes as potential factors, but there are not so many evidences to support this idea (Brin et al 2003). Other conditions like the presence of a trauma to one of the teeth before tretament starts, structure of the alveolar bone and its density, stage of root formations can influence the beginning of this type of resorption (Mohandesan 2007)

Orthodontic treatment means, generally speaking, to apply some forces to biological structures. Every single type of tissue (bone tissue, 
gingival tissue) has a particular way to respond to the action of orthodontic forces. Some authors found a correlation between these mechanical factors, such as the type of appliance used, type of force used for teeth movement (intrusion, extrusion), intensity of applied force and root resorption (Smale et al 2005, Pandis et al 2008 ).

In our study we found that there is a direct correlation between apical root resorption (especially grade 2) and the total amount of time when the appliances were on the teeth, although other studies show that treatment time is not related to this process (Lund et al 2011). Another important finding in this study is that the most affected teeth during this changes are incisors, bot in the upper and lower arch. This conclusion relates to finding in studies conducted by various researchers (Artun et al 2009, Hartsfield et al 2009) .

\section{Conclusion}

Within the limitations of this study, we conclude that there is a high correlation between the amount of apical root resorption and the duration of active fixed appliance treatment. The most affected teeth by this type of resorption in the upper arch and even in the lower arch, are incisors. Therefore, we recommend that a radiographic control should be carried out routinely. In patients wearing fixed appliance treatment exceeding 6 months, is recommended a follow-up interval of 6 months during treatment in order to observe if some modifications occur.

\section{References:}

Al-Qawasmi, Riyad A., et al. "Genetic predisposition to external apical root resorption in orthodontic patients: linkage of chromosome-18 marker." Journal of Dental research 82.5 (2003): 356-360.

Apajalahti, Satu, and Jaakko Sakari Peltola. "Apical root resorption after orthodontic treatment-a retrospective study." The European Journal of Orthodontics 29.4 (2007): 408-412.

Hartsfield, J. K., Eric T. Everett, and R. A. Al-Qawasmi. "Genetic factors in external apical root resorption and orthodontic treatment." Critical Reviews in Oral Biology \& Medicine 15.2 (2004): 115-122.

Segal, G. R., P. H. Schiffman, and O. C. Tuncay. "Meta analysis of the treatment-related factors of external apical root resorption." Orthodontics \& craniofacial research 7.2 (2004): 71-78.

Liou, Eric JW, and Peter MH Chang. "Apical root resorption in orthodontic patients with en-masse maxillary anterior retraction and intrusion with miniscrews." American Journal of Orthodontics and Dentofacial Orthopedics137.2 (2010): 207-212.

Dudic, Alexander, et al. "Detection of apical root resorption after orthodontic treatment by using panoramic radiography and cone-beam computed 
tomography of super-high resolution." American Journal of Orthodontics and Dentofacial Orthopedics 135.4 (2009): 434-437.

McNab, Scott, et al. "External apical root resorption following orthodontic treatment." The Angle orthodontist 70.3 (2000): 227-232.

Brezniak, Naphtali, and Atalia Wasserstein. "Orthodontically induced inflammatory root resorption. Part I: the basic science aspects." The Angle orthodontist 72.2 (2002): 175-179.

Brin, Ilana, et al. "External apical root resorption in Class II malocclusion: a retrospective review of 1-versus 2-phase treatment." American Journal of Orthodontics and Dentofacial Orthopedics 124.2 (2003): 151-156.

Mohandesan, Hooman, Hossein Ravanmehr, and Nasser Valaei. "A radiographic analysis of external apical root resorption of maxillary incisors during active orthodontic treatment." The European Journal of Orthodontics29.2 (2007): 134-139.

Smale, Isolde, et al. "Apical root resorption 6 months after initiation of fixed orthodontic appliance therapy." American journal of orthodontics and dentofacial orthopedics 128.1 (2005): 57-67.

Pandis, Nikolaos, et al. "External apical root resorption in patients treated with conventional and self-ligating brackets." American journal of orthodontics and dentofacial orthopedics 134.5 (2008): 646-651.

Lund, Henrik, et al. "Apical root resorption during orthodontic treatment: a prospective study using cone beam CT." The Angle orthodontist 82.3 (2011): 480-487.

Årtun, Jon, et al. "Identification of orthodontic patients at risk of severe apical root resorption." American Journal of Orthodontics and Dentofacial Orthopedics135.4 (2009): 448-455.

Hartsfield Jr, J. K. "Pathways in external apical root resorption associated with orthodontia." Orthodontics \& craniofacial research 12.3 (2009): 236242. 